method. While the average bending strength of the untreated alloy was 245 $\mathrm{MPa}$, the average bending strength of the alloy treated with the magnetic field was $430 \mathrm{MPa}$, roughly a $75 \%$ increase. The elastic modulus remained unchanged. Scanning electron microscopy observations of the fracture surfaces of the treated specimens showed typical ductile fracture characteristics. The fracture surfaces of the untreated specimens clearly showed brittle characteristics with microcracks, the researchers reported.

In order to understand the underlying mechanism, C.W. Wu in the Department of Mechanical Engineering at Dalian said that the research group performed electron probe microanalysis on the untreated and treated samples. The untreated alloy consists of $\mathrm{NiAl}, \mathrm{Cr}(\mathrm{Mo})$, and Heusler phases. These do not change when the alloy is heated to $900^{\circ} \mathrm{C}$. In the magneticfield-treated samples, the Heusler phases at the $\mathrm{NiAl} / \mathrm{Cr}(\mathrm{Mo})$ grain boundaries were partly dissolved into the NiAl and $\mathrm{Cr}(\mathrm{Mo})$ phases. In addition, small Heusler phase particles at the $\mathrm{Cr}(\mathrm{Mo})$ and matrix phase interfaces partially dissolved into the NiAl matrix (see Figure 1). The researchers speculated that the dissolution of the Heusler phase due to the highmagnetic-field treatment contributed to the improvement in bending strength and ductility of the alloy. The exact mechanism, however, remains unclear.

GOPAL RAO

\section{Metalorganic Building Block Enables Synthesis of Four Supramolecular Isomers}

While supramolecular isomerism is widely encountered in the field of crystal engineering, the majority of these that have been reported require a co-existence of different guest components for production. An elementary system of supramolecular isomers that has been encountered is the zero-dimensional ring-like, one-dimensional zigzag, and helical chains that can be derived from a stoichiometry of 1:1 metal/ligand molar ratio. Now, X.-M. Chen and colleagues from Sun Yat-Sen University in China have fabricated low-dimensional supramolecular isomerism (chairs, zippers, and chains) of $0 \mathrm{D} / 1 \mathrm{D}$ coordination polymers without the use of guest components.

By using a binary $\mathrm{Cu}^{+1}$ triazolate $\left[\mathrm{Cu}^{+1}(2-\mathrm{Hpytz})\right]_{x}(2-\mathrm{Hpytz}=3,5$-di-(2pyridyl)-1,2,4-triazolate) pre-designed, metalorganic building block, the researchers isolated four types of air-stable crystals of different colors and/or shapes. Crystallographic studies revealed the stoichiometry of 1:1 (metal/ligand) for all

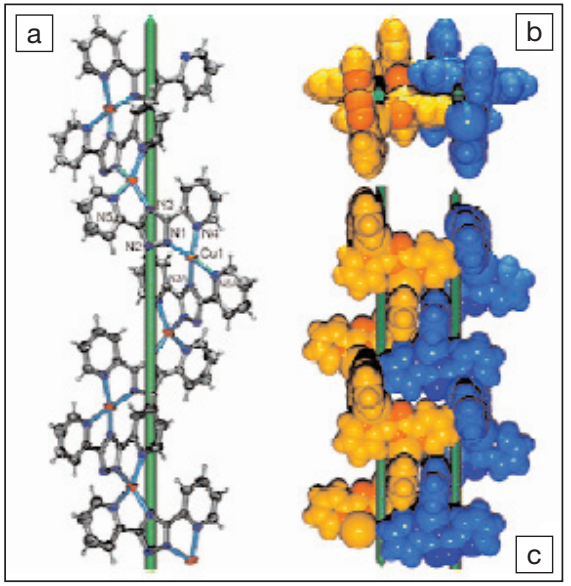

Figure 1. ORTEP drawing (50\% A: 0.5 $\mathrm{y}, \mathrm{x}-0.5, \mathrm{z}-0.25)$ of a single 41 helix in Complex IV (a). Top (b) and side (c) views of two adjacent helical chains in space-filling modes. ORTEP stands for Oak Ridge thermal-ellipsoid plot program. Reproduced with permission from Chemical Communications (2005), p. 1258; DOI: 10.1039/b416095a.

(C) 2005 Royal Society of Chemistry.

complexes isolated. The researchers' findings were reported in the March 1 issue of Chemical Communications (p. 1258; DOI: 10.1039/b416095a).

The different complexes were synthesized based on the different conformations of the coordinating ligands. Complex I was a red polyhedral crystal that possessed a centrosymmetrical, chair-like tetrameric superstructure. The researchers attributed this superstructure to the cis-cis and cis-trans 2-Hpytz ligand conformations. Complex II (with the conformation of Hpytz ligand, cis-trans, and half a cis-cis) was a red column crystal that has a highly distorted tetrahedral environment that produced a zipper-like double-chain superstructure. Complex III (with the conformation of pytz ligand, one cis-trans, and a distorted tetrahedral $\mathrm{Cu}^{+1}$ metal center) was an orange column crystal that is centrosymmetric, which gave rise to anti-parallel, aligned, polar zigzag chains, the researchers said.

Complex IV (see Figure 1) was an orange needle-like crystal that gave rise to a homochiral structure with an antiparallel, aligned, single-stranded $4_{1}$ helix, which is rare, the researchers said. This complex was synthesized by varying the reaction times and conditions. The researchers said that this system represents the first example of low-dimensional, supramolecular isomerism in coordination polymers and gives rise to controlled crystallization of individual isomers.

LARKEN E. EULISS

\section{Wavelength Multiplexing of Microsphere Resonators Used to Characterize Nanolayers}

Biosensors are widely used in clinical and military applications as well as for the characterization of biomolecular interactions important in drug discovery. While traditional optics employ nanolayers made from inorganic insulators, semiconductors, and metals, the emerging field of biophotonics employs DNA, proteins, lipids, and hydrogels in soft condensed biofunctional layers formed at aqueous-solid interfaces. Recently, S. Arnold and co-researchers from Polytechnic University in New York have developed a non-invasive method to optically characterize bioactive nanolayers formed in situ on a silica microsphere.

As reported in the March 1 issue of Optics Letters (p. 510), the research team developed an appropriate theory, validated it experimentally, and then demonstrated their method's utility by optically characterizing a biophysically relevant hydrogel. Focusing on the theoretical effect that a nanolayer has on a microsphere's whispering-gallery modes (WGMs), the researchers applied perturbation theory to transform the electricfield equations for the transverse electric WGMs to make the problem analogous to the solution of the Schrödinger equation with an appropriate effective potential. The optical transmission spectra of the WGMs have resonances that are perturbed by the addition of nanolayers of material. By judiciously choosing two wavelengths, $\lambda_{1}$ and $\lambda_{2}$ (760 nm and 1310 $\mathrm{nm}$, respectively), the researchers found that the ratio $S$ of the fractional shifts in the resonances of the WGM wavelengths has the limit of $\lambda_{1} / \lambda_{2}(0.58$, in this case) for a thick layer and a limit approaching unity for an ultrathin layer. In addition, using two equations derived by the researchers, both the layer thickness and the optical dielectric constant can be independently determined from the measurements of the wavelength shifts of the WGMs.

The researchers conducted two experiments to test these limits. In the first, as a monolayer of bovine serum albumin $(\sim 3$ $\mathrm{nm}$ thick) was formed on a silica microsphere, monitoring of the resonances associated with the two wavelengths showed that each shifted about the same amount, $S=1$, consistent with the theory for a thin layer on the microsphere. In the second test case, the researchers injected $\mathrm{NaCl}$ into the water surrounding a microsphere and incrementally increased the salt concentration by $0.1 \mathrm{M}$. A plot of the resonance shift at $\lambda_{1}$ versus the resonance shift of $\lambda_{2}$ displayed a slope of 0.54 , consistent with that predicted for the for- 
mation of a thick layer around the microsphere. The researchers said that both limiting tests agree reasonably with theory.

The researchers then characterized a nanolayer composed of poly-L-lysine hydrogel, which, due to its very high charge, readily adsorbs negatively charged biomolecules. Poly-L-lysine hydrogel nanolayers are also difficult to characterize using other methods because it forms such thin layers with low contrast to water. The researchers observed a very small resonance shift, which their theory indicates corresponds to a layer thickness of $110 \mathrm{~nm}$ and an excess refractive index of 0.0012 . The researchers said that as a result of their method, "the WGM resonator goes beyond its original promise as a biosensor." In addition, the researchers anticipate that real-time measurement of $S$ will reveal morphology changes concomitant with increases in layer density. Furthermore, Arnold and his co-researchers said that an alternate formulation of their theory applied to nonspherical particles "shows promise for looking at heterogeneous structures such as adsorbed bacteria."

STEVEN TROHALAKI

\section{Metalorganic Gel Used for Porous Organic Polymer Template}

Coordination polymers where metal centers are linked by organic bridging ligands represent novel materials with potentially useful porosity and inclusion properties. Much of the research on this class of materials has, however, focused on studying single crystals of these materials to understand the molecular basis for their formation and properties. Polymer gels-which potentially have interesting properties in catalysis, sensing, and as responsive materials - have been far less studied. In the March 28 issue of Chemical Communications (DOI: $10.1039 / \mathrm{b} 418554 \mathrm{~d}), \mathrm{Q}$. Wei and S.L. James from the Queen's University of Belfast in Northern Ireland report the reaction of iron nitrate and 1,3,5-benzenetricarboxylic acid in ethanol to give a metalorganic gel with a solvent content of $95-98 \%$ by weight.

The researchers propose that coordination polymer particles are formed by rapid cross-linking polymerization between $\mathrm{Fe}^{3+}$ and the benzenetricarboxylic acid and that these particles are further crosslinked to provide macropores that trap solvent molecules. The gel can be formed in the presence of organic monomers, which can be trapped in the cavities and polymerized to form porous imprints of the metalorganic gel. Wei and James demonstrate this for poly(methyl methacrylate) (PMMA) by polymerizing methyl methacrylate in the gel cavities and dissolving the gel framework in hydrochloric acid. The resulting PMMA imprint contains disordered pores with a size range of $1-10 \mu \mathrm{m}$.

James said that the major advantage of this technique is that it is a straightforward and inexpensive route to templating porous organic polymers, which have potential applications in supports and separations. The presence of metalorganic particles within the polymer matrix suggests that other interesting magnetic and responsive properties may be observable in these materials, the researchers said.

SARBAJTT BANERJEE

\section{Tungsten Nanoparticles Embedded in Silica \\ Enhance Nonvolatile Memory}

Many consumer electronic products use nonvolatile memory devices in their operation. One such device is electrically erasable and programmable read-only memory (EEPROM), which uses a floating gate structure. In the drive to develop memories with lower power consumption and faster erase/write cycles, researchers have begun to incorporate nanocrystals into their EEPROMs in order to improve performance. Recently, T.C. Chang of the National Sun Yat-Sen University, P.T. Liu of the National Chiao Tung University, and their colleagues demonstrated that replacing the floating gate in EEPROMs with tungsten nanocrystals in a silica matrix reduces the operating voltage and increases endurance. Using nanoparticles in
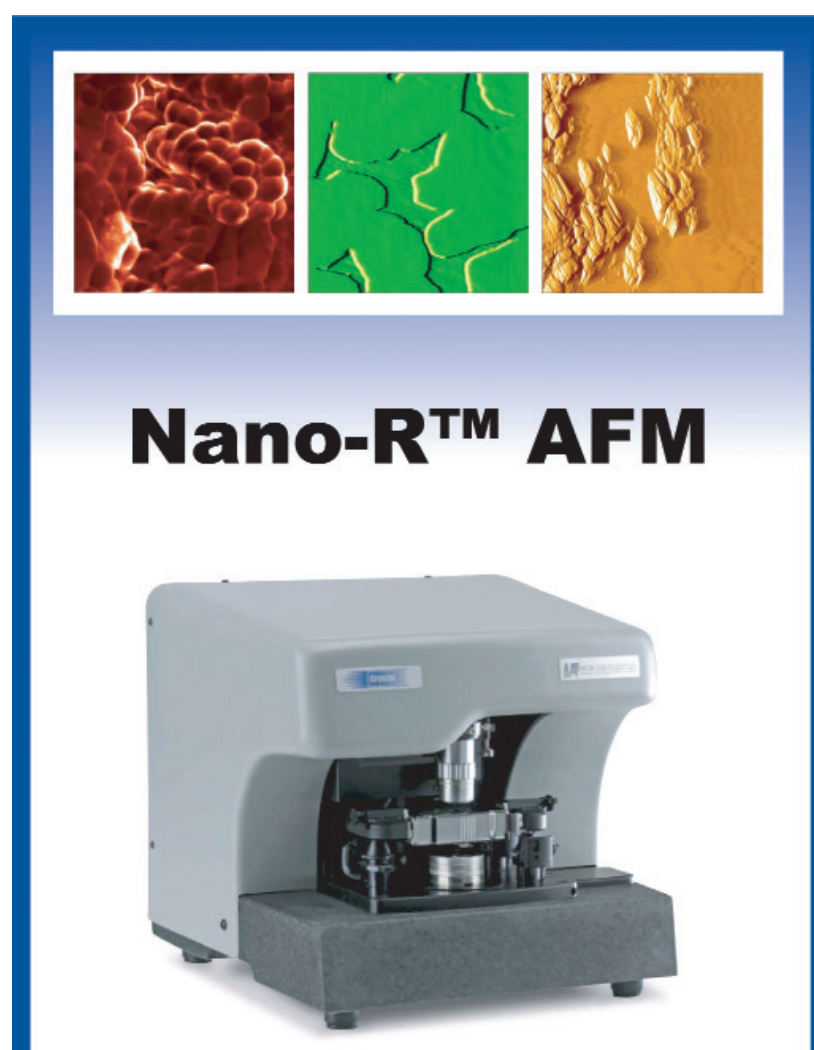

- High Performance

- Easy to Use

- Versatile

Nano- $R^{\mathrm{TM}}$ is available with a traditional light lever scanner and the advanced Crystal Scanner ${ }^{\mathrm{TM}}$.

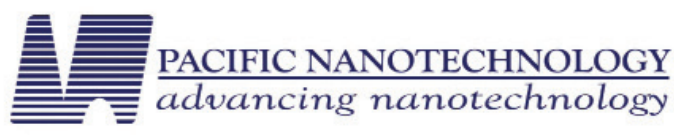

3350 Scott Blvd. \#29

Santa Clara, CA 95054

408-982-9492

www.pacificnanotech.com

www.probestore.com
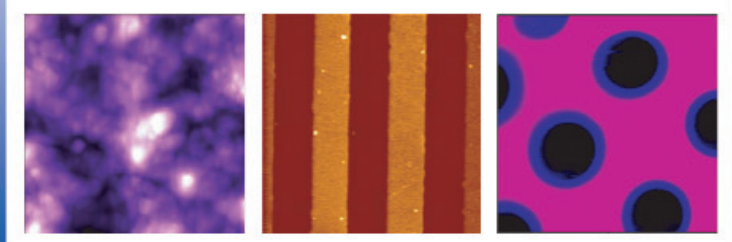

For more information, see http://advertisers.mrs.org 